\title{
Preface
}

\section{Chest Drainage Management: Where Are We Now?}

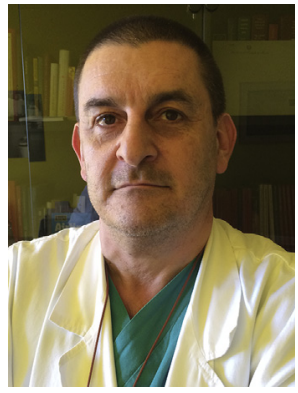

Pier Luigi Filosso, MD, FECTS

Editor

This issue of Thoracic Surgery Clinics is dedicated to the management of chest drains.

Chest drain insertion is one of the most common surgical procedures performed in routine clinical practice. It is usually performed by thoracic surgeons but, oftentimes, also by emergency physicians, intensivists, pulmonologists, interventional radiologists, and nonphysician advanced practitioners in the emergency setting. Despite the many benefits, this procedure is not devoid of complications, and potential significant morbidity and mortality may exist. Furthermore, its management is usually strictly dependent on the physician's personal preference, since up to now, univocal guidelines did not exist.

This issue's review articles cover most of what physicians face when treating a patient with a chest tube, and have been written by worldrenowned experts in this field.

I am deeply indebted to all of the authors who contributed to the realization of this issue, as well as the Elsevier staff, particularly John Vassallo and Susan Showalter, with whom I had long discussions that helped me in the finalization of this issue.

Finally, I wish to dedicate this issue of Thoracic Surgery Clinics to all those women and men surgeons who, through their hard work, daily commitment, and devotion to the field of thoracic surgery help to promote and progress our specialty, ensuring patients a high quality of care and a better quality of life, and improving their long-term survival. 